\title{
Scalable attoliter monodisperse droplet formation using multiphase nano-microfluidics
}

\author{
Lingling Shui $\cdot$ Albert van den Berg • \\ Jan C. T. Eijkel
}

Received: 9 December 2010/ Accepted: 21 January 2011/Published online: 12 February 2011

(C) The Author(s) 2011. This article is published with open access at Springerlink.com

\begin{abstract}
We demonstrate a robust method to produce monodisperse femtoliter to attoliter droplets by using a nano-microfluidic device. Two immiscible liquids are forced through a nanochannel where a steady nanoscopic liquid filament forms, thinning close to the nanochannel exit to a microchannel due to the capillary focusing. When the nanoscopic filament enters the microchannel, monodisperse droplets are formed by capillary instability. In a certain range of physical parameters and geometrical configurations, the droplet size is only determined by the nanochannel height and independent of liquid flow rates and ratios, surfactants, and continuous phase viscosity. By using nanochannels with a height of $100-900 \mathrm{~nm}$, 0.4-3.5 $\mu \mathrm{m}$ diameter droplets (volume down to $30 \mathrm{aL}$ ) have been produced. The generated droplets are stable for at least weeks.
\end{abstract}

Keywords Nano-microfluidics · Droplet · Attoliter . Monodisperse $\cdot$ Capillary focusing

\section{Introduction}

Microfluidics has received enormous attention because of the ready availability of fabrication and measurement methods (Losey et al. 2002; Tien et al. 2002; Sinton 2004;

Electronic supplementary material The online version of this article (doi:10.1007/s10404-011-0776-7) contains supplementary material, which is available to authorized users.

L. Shui $(\bowtie) \cdot$ A. van den Berg $\cdot$ J. C. T. Eijkel BIOS/Lab-on-a-Chip Group, MESA + Institute for Nanotechnology, University of Twente, P.O. Box 217, 7500 AE Enschede, The Netherlands

e-mail: L.Shui@utwente.nl van den Berg and Lammerink 1998). Many areas employ multiphase droplet-based microfludics (Shui et al. 2007; Gunther and Jensen 2006), including inkjet printers (van Dam and Le Clerc 2004), systems for separation of biochemical samples (Fujimura et al. 2003), manipulation of biomolecules (Lo et al. 2004), bio-sensing (Wang et al. 2006), single cell analysis (Yong et al. 2010), enhanced mixing for bio-sample reactions (Yang et al. 2006), biomolecular detection (Tseng et al. 2004), drug delivery devices (Chung et al. 2008), dairy analysis (Skurtys and Aguilera 2008), microelectronic cooling (Nilson et al. 2006), explosives detection (Piorek et al. 2007), bubble computing (Prakash and Gershenfeld 2007), interfacial tension measurement (Xu et al. 2008), and analysis of emulsions, foams, and bubble coalescence (Kralj et al. 2005). As general characteristics, the droplets should be as stable, monodisperse, reproducible, and controllable as possible.

Precise understanding of biological or chemical functions requires the ability to isolate and study single molecules. Although measurements can be made on single molecules in a dilute solution as they diffuse through the measurement volume, most such approaches rely on sophisticated optical strategies to limit the detection volume. Small containers can provide a means of confining single molecules inside of a measurement volume. We will need attoliter (or femtoliter) size containers to study single enzyme molecule activity with a $\mu \mathrm{M}$ (or $\mathrm{nM}$ ) solution. The contents of individual containers should if possible be independently controllable so that distinct reactions can take place in separate containers. An emulsion in which one liquid forms droplets in another immiscible liquid (water-droplet-in-oil or oil-droplet-in-water) is a simple and attractive way of creating such containers ( $\mathrm{Lu}$ et al. 1998; Nakano et al. 2003). 
Microdroplets can be formed using flow-focusing, either by increasing shear gradients or by drawing the stream into a thin filament that breaks up by the Rayleigh-Plateau instability (Anna et al. 2003). Droplets generated in microfluidic devices are commonly created by squeezing or shearing the dispersed liquid (oil or water) into the continuous liquid (water or oil). Droplet size depends on microchannel geometry (Dollet et al. 2008; Sugiura et al. 2002b; Ravigururajan 1998; Link et al. 2004), flow rates (Garstecki et al. 2006; Martín-Banderas et al. 2005; Utada et al. 2007), and fluid properties (Ganan-Calvo and RiescoChueca 2006; Tice et al. 2004; Ganan-Calvo et al. 2004; Lam et al. 2002). Many parameters make the droplet formation process in such cases complex and sensitive to various environmental influences. Furthermore, the droplet size is limited by the microfabricated channel size (typically, in the micrometric range). Therefore, a method is required which can not only produce monodisperse attoliter droplets, but is also stable and robust, and inertial to small changes in the flow rate.

One of the methods to create droplets is by forming a thin liquid filament, which then spontaneously breaks up due to the capillary instability. The formation of a liquid filament is then a mandatory preliminary step for producing a droplet (Chen et al. 2003). This procedure is widely applied in flow-focusing devices. In these devices, an inner liquid thread can be created by outer liquid shearing (Ganan-Calvo and Gordillo 2001; Amyot and Plouraboue 2007; Garstecki et al. 2004; Gordillo et al. 2001; Barrero et al. 1998; Utada et al. 2007; Ganan-Calvo 1998). In this case, however, the confinement is not stable and can easily be disturbed by dynamic fluctuations.

A stable and strong confinement of a liquid filament, on the other hand, can be obtained by solid structures (nanochannels or nanoholes). Since this confinement stabilizes the liquid thread, breakup does not easily occur. When the confinement is subsequently removed by a sudden increase of channel diameter, capillary instability will cause formation of monodisperse droplets (van Dijke et al. 2010; Malloggi et al. 2010; van der Zwan et al. 2009, Sugiura et al. 2002a, b). Here, we investigated the droplet formation of this method and the scalability of this approach to produce droplets with a volume of less than $100 \mathrm{aL}$ droplets (diameter below $600 \mathrm{~nm}$ ) by using nanochannel sections down to $100 \mathrm{~nm}$.

\section{Experimental}

The channel structure used for the experiments is shown in Fig. 1. The volume flow in the nanochannel section is regulated by the entire channel structure. For the experiments described in this study, the nano-microfluidic interface is of prime importance, consisting of two identical inlets, a constriction channel and a microchannel. The inlets and constriction channel are nanochannels with dimensions of height $(h)=100-900 \mathrm{~nm}$, width $(w)=10$, 20 , or $50 \mu \mathrm{m}$ and length $(l)=500$ or $1000 \mu \mathrm{m}$; and the microchannel dimensions are: height $(H)=10 \mu \mathrm{m}$, width $(W)=50$ or $100 \mu \mathrm{m}$, length $(L)=5 \mathrm{~mm}$.

The devices were fabricated using standard photolithographic techniques. Nanochannels and microchannels were etched into a borosilicate glass wafer. The connection holes were drilled in a second borosilicate glass wafer using powder blasting techniques. Subsequently, these two wafers were aligned and thermally bonded together. We diced the bonded wafers to $10 \times 20 \mathrm{~mm}$-sized chips, which were mounted in a home-made chip holder and connected to gastight syringes (Microliter Syringes, Hamilton) via Nanoport connectors (Upchurch Scientific). Harvard syringe pumps (PHD 22/2000, Harvard Apparatus) were used to drive the liquid flow. The two-phase flow was visualized by an inverted microscope (Leica DMIRM) and recorded using a CCD camera (Orca ER).

The water phase was made fluorescent (appearing white in the microphotographs) by dissolving fluorescein sodium salt (Sigma-Aldrich Chemie GmbH, Germany) in de-ionized water at a final concentration of $0.01 \mathrm{~mol} \mathrm{~L}^{-1}\left(\eta_{\mathrm{w}}=\right.$ $1 \mathrm{mPa} \mathrm{s}$ ). The organic phase (dark in the images) consisted of hexadecane (Sigma-Aldrich Chemie GmbH, Germany) without any added fluorescent markers $\left(\eta_{\mathrm{o}}=3 \mathrm{mPa} \mathrm{s}\right)$. Glycerol (Sigma-Aldrich Chemie GmbH, Germany) has been used to tune the water phase viscosity from 1 to $10 \mathrm{mPa} \mathrm{s}(60 \mathrm{wt} \%)$. Surfactants have been used to stabilize generated droplets. The surfactants used were sodium

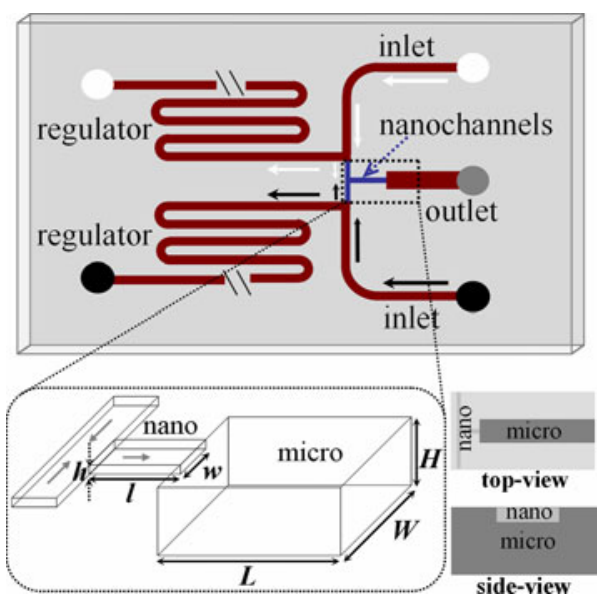

Fig. 1 Sketch of the nano-micro fluidic chip design. Fluids are introduced from the inlet, and split to nanochannel and the regulator microchannel at the junction of inlet, regulator and nanochannel. In the nanochannels, two immiscible liquids flow head-on and meet at the junction and then step from the nanochannel to the microchannel outlet 
dodecyl sulfate $\left(\mathrm{CH}_{3}\left(\mathrm{CH}_{2}\right) 11 \mathrm{OSO}_{3} \mathrm{Na}\right.$, SDS $99+\%$, Sigma-Aldrich Chemie GmbH, Germany), Tween80 (Sigma-Aldrich Chemie GmbH, Germany), and Span80 (Sigma-Aldrich Chemie GmbH, Germany). Solutions were prepared by dissolving hydrophilic surfactants (0.01 M SDS and $1 \mathrm{wt} \%$ Tween80) in water or hydrophobic surfactant (1 wt\% Span80) in hexadecane. The surfactant concentrations were in all cases above the critical micelle concentrations. Solutions were degassed under vacuum for $1 \mathrm{~h}$ before using. All chemicals were used directly as received without further treatment. If it is not mentioned specifically, the two phases used are SDS $(0.01 \mathrm{M})$ fluorescein $(0.01 \mathrm{M})$ aqueous solution and hexadecane.

\section{Results and discussion}

\subsection{Droplet formation at the nanochannel-} microchannel interface

The liquid flow in the nanochannels is controlled using a syringe pump. The complications of conventional syringe pumping at ultralow flow rates $\left(<10^{-2} \mu \mathrm{L} \mathrm{min}{ }^{-1}\right)$ were prevented by designing an integrated internal flow control system to create a flow split between a microchannel and the nanochannel, as previously reported by us and the group of Kitamori (Tamaki et al. 2006; Hibara et al. 2009; Shui et al. 2009a). The entire chip design is shown in Fig. 1. Liquids are introduced to the nanochannels (blue) from the sides by microchannels (red) which are connected between an inlet and a flow regulating channel. The total flow is divided between the nanochannel and the flow regulating microchannel, with a flow split ratio which is inversely proportional to their flow resistance ratio (Shui et al. 2009b). By designing the fluidic network, we could therefore control the flow in the nanochannel as required. We obtained flow rates of $10^{-5}-10^{-3} \mu \mathrm{L} \mathrm{min}{ }^{-1}$ in the nanochannel by using a typical syringe pump with a flow rate of $0.1-10 \mu \mathrm{L} \mathrm{min}{ }^{-1}$ (split ratio on average $1: 10^{4}$ ).

In the nanochannel area, oil and water met at the junction and flowed together in the constriction nanochannel. In the constriction nanochannel, the oil phase flowed either as large droplets (Fig. 2a) or as a thread parallel to water (Fig. 2b). When the oil phase (large droplets or thread) exited from the nanochannel into the microchannel, it spontaneously broke up into monodisperse oil-in-water droplets (see the online movie1 and movie2).

The droplet formation mechanism at devices that employ a sudden expansion of the flow channel has been described in several recent papers (van Dijke et al. 2010; Malloggi et al. 2010; Sugiura et al. 2002a, b). To summarize, the oil phase moves through the inlet nanochannel as a wide filament when the hydrodynamic pressure of oil is sufficiently large

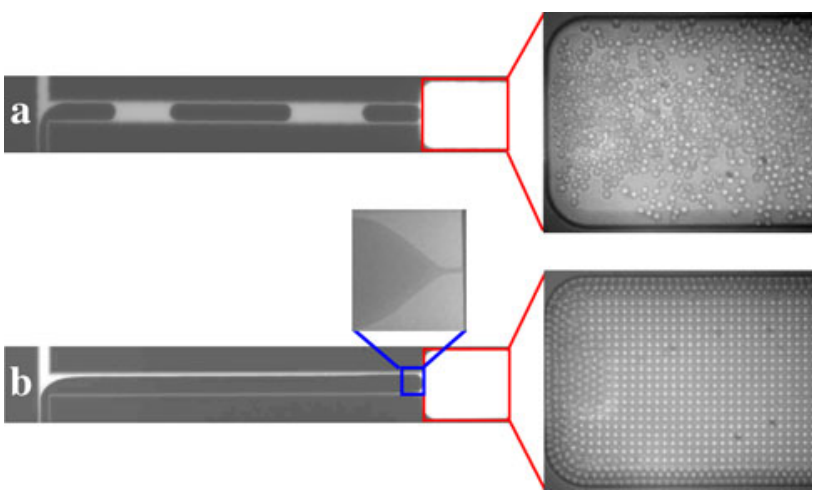

Fig. 2 Snapshots of the droplet formation at the nanochannelmicrochannel interface. a Oil droplets generated at the junction split to smaller droplets at the nanochannel-microchannel interface. b Oil flows parallel with water in the nanochannel and forms droplets at the nanochannel-microchannel interface. The device dimensions are: $h=520 \mathrm{~nm}, w=20 \mu \mathrm{m}, l=500 \mu \mathrm{m}, H=10 \mu \mathrm{m}$, and $W=50 \mu \mathrm{m}$

compared to the interfacial tension stress acting at the oilwater interface. The filament width remains almost constant along the constriction nanochannel up to a point close to the interface where the oil tip shrinks to a narrow filament. This capillary focusing as recently described (Malloggi et al. 2010) (see Figs. 3, 4) has its origin in the sudden decrease of the capillary pressure by approximately $2 \sigma / h$ (with $\sigma$ the interfacial tension and $h$ the nanochannel height) when the oil filament expands in the microchannel. This pressure drop causes an acceleration of the oil phase close to the interface with the microchannel, leading to a filament thinning. Measurements made of the droplet formation with a fast camera (Fig. 3) show a gradual narrowing of the oil filament
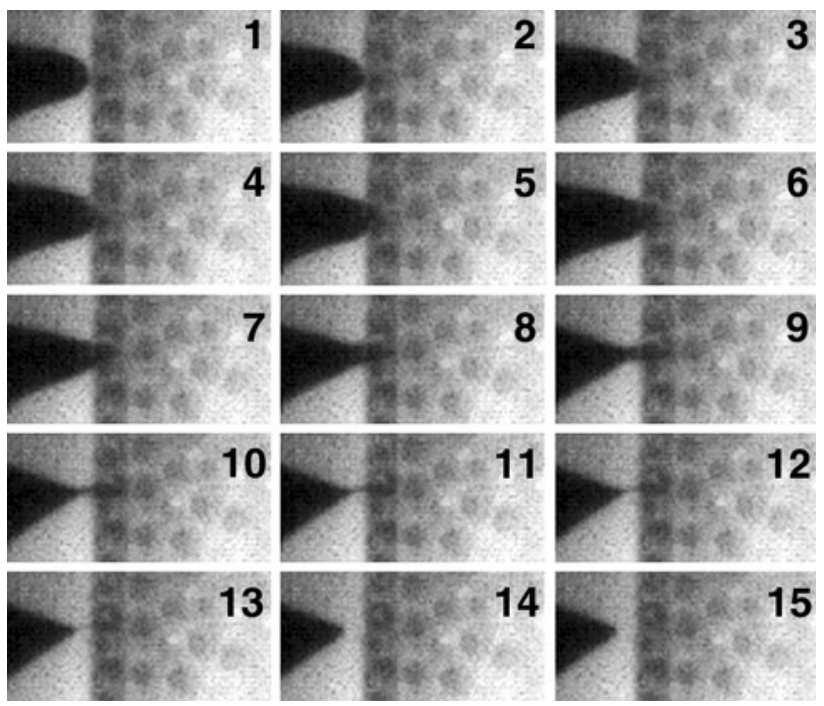

Fig. 3 1-15 Droplet formation process. Time interval between images is $0.2 \mathrm{~ms}$. Nanochannel height $h=520 \mathrm{~nm}, Q_{\mathrm{o}}=2.5$,

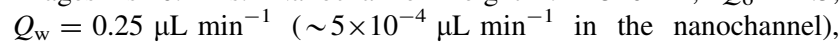
droplet diameter is $2.5 \mu \mathrm{m}$ and droplet formation frequency is $100 \mathrm{~Hz}$ 


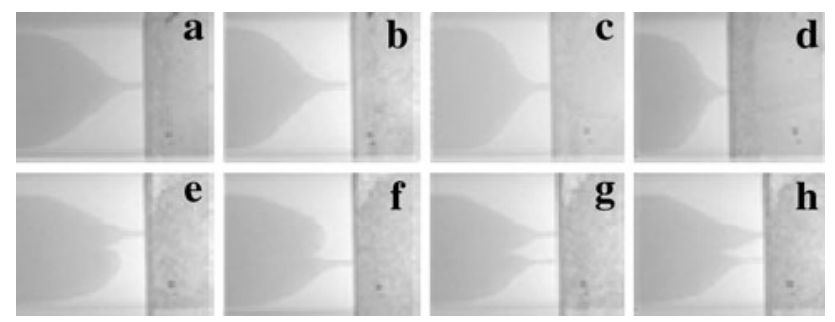

Fig. $4 a-h$ Filament shapes at different flow rates in nanochannels:

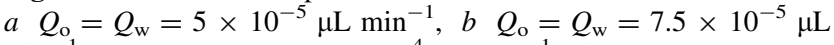

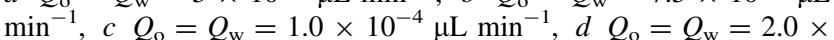
$10^{-4} \mu \mathrm{L} \mathrm{m^{-1 }}, e-h$ Formation of two filaments as occasionally occurring; $Q_{\mathrm{o}}=5 \times 10^{-5} \mu \mathrm{L} \min ^{-1}$ and $Q_{\mathrm{w}}=3 \times 10^{-5} \mu \mathrm{L} \mathrm{min}{ }^{-1}$. The device dimensions are: $h=260 \mathrm{~nm}, w=50 \mu \mathrm{m}, l=500 \mu \mathrm{m}$, $H=10 \mu \mathrm{m}, W=100 \mu \mathrm{m}$, and $L=5 \mathrm{~mm}$

neck and subsequent snap-off due to the insufficient supply of oil to the expanding droplet in a way comparable to the simulations (van Dijke et al. 2008). Snap off was seen to occur in about $1 \mathrm{~ms}$ in a 520-nm high channel. Qualitatively, we found that the oil filaments were longer and narrower in shallower nanochannels.

Filaments occurring at different flow rates are shown in Fig. 4a-d. The length of the thinned filament decreases with increasing oil flow rate. Typically, we also found that the thin oil filaments were more prominent (longer and sharper) in shallower nanochannels. When a perturbation was applied to the tip, for example by a small obstruction in the constriction channel, the oil tip could split into two filaments (symmetric or asymmetric) close to the nanochannel exit (Fig. 4e-h). Same sized droplets were observed to break up from these two oil filaments.

\subsection{The dependence of the droplet size} on the nanochannel height

When an unconfined steady liquid filament (thread) spontaneously breaks up, the droplet size is proportional to the filament size in a certain range of experimental parameters (Ganan-Calvo and Gordillo 2001; Ganan-Calvo 1998; Utada et al. 2007). For the breakup of confined threads on expansion in a larger channel, it has been found in microdevices that the droplet size is proportional to the dimension of the confining structure (van Dijke et al. 2010; Malloggi et al. 2010; van der Zwan et al. 2009). Here we created nanoconfinements of the oil threads by employing devices with nanochannel heights $h$ of 100,160, 260, 520, and $900 \mathrm{~nm}$; widths $w$ of 10,20 , and $50 \mu \mathrm{m}$; and lengths $l$ of 500 and $1000 \mu \mathrm{m}$. The droplet diameter was optically measured in the microchannel section where the droplets are spherical.

Figure 5a-d show the droplets created in the devices with different nanochannel height. Figure 5e shows the droplet size as a function of flow rate in different devices. At a constant height nanochannel, over a wide range of flow conditions, typically for $Q_{\mathrm{o}}=Q_{\mathrm{w}}<10^{-3} \mu \mathrm{L} \mathrm{min}^{-1}$ in the devices, the observed oil droplets were of constant size and monodisperse. However, the droplet size increased with flow rate when the flow rate increased over a threshold $\left(Q>10^{-3} \mu \mathrm{L} \mathrm{min}{ }^{-1}\right)$. Error bars in Fig. 5e indicate the standard deviation of the measured diameters. In the flowrate dependent regime, a slight increase in polydispersity was observed. Smaller (shallower) nanochannels showed wider flow rate operating ranges. It was found that the nanochannel width and length had almost no effect on the droplet size in the devices; however, the droplet size was found to be linearly proportional to the nanochannel height with a proportionality factor determined of 4 , as indicated in Fig. 5f. The smallest droplets were produced using the devices with the nanochannel height of $100 \mathrm{~nm}$. Linear extrapolation of the droplet diameters obtained in larger channels yields a droplet diameter of $400 \mathrm{~nm}$ (in the $h=100 \mathrm{~nm}$ device), which would imply a droplet volume of approximately $30 \mathrm{aL}$ (see Fig. 5). At this droplet
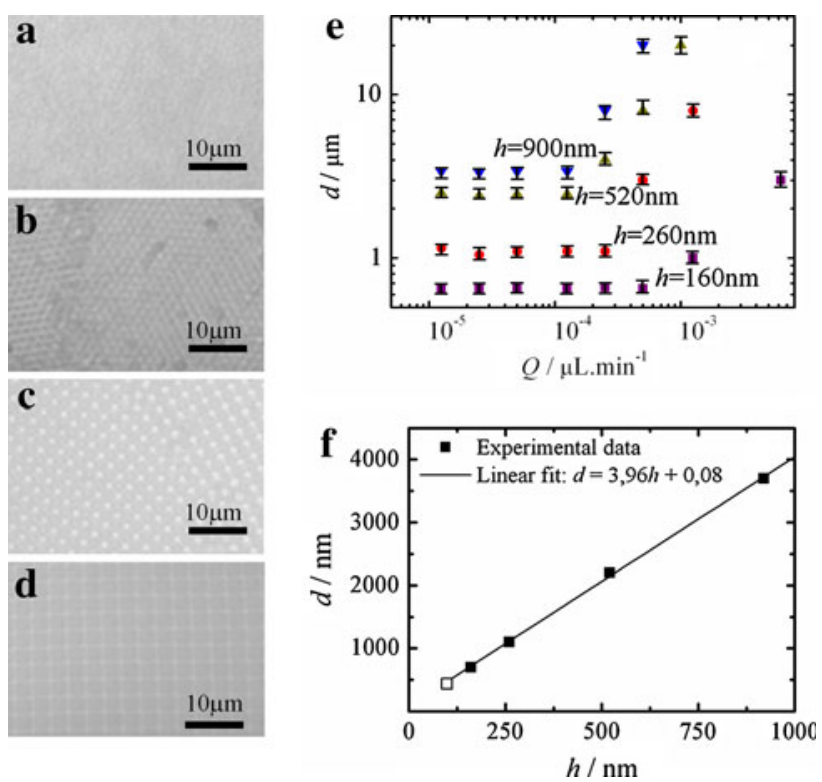

Fig. 5 The microphotographs of monodisperse droplets in different devices: a $h=160 \mathrm{~nm}$, b $h=260 \mathrm{~nm}$, c $h=520 \mathrm{~nm}$, and $\mathbf{d} h=$ $900 \mathrm{~nm}$. The images were taken using transmitted light. e The oil droplet diameter as a function of flow rates at $Q_{\mathrm{o}}=Q_{\mathrm{w}}$. The standard deviation of the droplet diameter was optically determined (instrumental error about $150 \mathrm{~nm}$ ). In the flow-rate independent regime, only the larger droplet $(3.5 \mu \mathrm{m})$ yielded a slightly larger standard deviation. The droplets in the flow-rate dependent regime showed larger standard deviations. f The droplet diameter versus nanochannel height in the platform region. The droplets formed in $h=100 \mathrm{~nm}$ devices are visible under fluorescence microscopy; however, their diameter is derived from the extrapolation of the linear data fit and indicated as $400 \mathrm{~nm}$ 
volume, single molecule experiments could be performed at conveniently preparable analyte concentrations of $50 \mu \mathrm{M}$.

We have also investigated the influence of the water phase viscosity (in the range of $1-10 \mathrm{mPa}$ s by addition of glycerol) and of the use of different surfactants (SDS, Tween80, and Span80), resulting in a surface tension in the range of $10^{-4}-10^{-2} \mathrm{~N} \mathrm{~m}^{-1}$ ) (Shui et al. 2009c) on the droplet formation process, to find out whether this method is sensitive to slight changes in fluidic properties. We did not observe a significant change of droplet size with water phase viscosity and nature of surfactants in the platform regime. However, the transition values from the platform region to the flow-rate dependent region were influenced by the relative liquid viscosities and surfactants. The transition occurs at lower flow rates if the water (continuous phase) viscosity increases. When using different surfactants, the transition occurred at lower flow rate in the order Span $80>$ Tween $80>$ SDS. Typically, the frequency of droplet formation in the devices ranged from 10 to $10^{4} \mathrm{~Hz}$, dependent on the flow rate. In future, the droplet production rate could be increased by using parallel nanochannels.

\section{Conclusions and outlook}

We have demonstrated the production of monodisperse attoliter droplets using nano-microfluidic devices. Over a wide range of absolute and relative flow rates, highly stable and monodisperse small $(0.4-3.5 \mu \mathrm{m}$ diameter) droplets were obtained in devices with nanochannel heights of 100-900 nm. The shallower nanochannels furthermore demonstrated wider flow rate operating ranges. The droplet size was linearly related to the nanochannel height. In this range, we did not observe obvious effect of liquid flow rates, surfactants and continuous phase viscosity on generated droplet size. Thus, we can predict droplet size generated in an existing device or design such a device to create droplets as required. Limitations to the obtainable droplet size will be posed by nanofabrication technology. The attoliter droplets can be used as small containers which will on average encapsulate one molecule per droplet at analyte concentrations in the $\mu \mathrm{M}$ range, forming a promising tool for single molecular studies. By using this method, we can further scale-down the droplet size by using shallower nanochannels, and scale-up the droplet production by using parallel nanochannels.

Acknowledgments This research was supported by the Dutch Ministry of Economic Affairs through a Nanoimpuls grant. The authors gratefully acknowledge Wim van Hoeve and Chao Sun for supporting with high speed camera.
Open Access This article is distributed under the terms of the Creative Commons Attribution Noncommercial License which permits any noncommercial use, distribution, and reproduction in any medium, provided the original author(s) and source are credited.

\section{References}

Amyot O, Plouraboue F (2007) Capillary pinching in a pinched microchannel. Phys Fluids 19(3):033101

Anna SL, Bontoux N, Stone HA (2003) Formation of dispersions using "flow focusing" in microchannels. Appl Phys Lett 82(3): 364-366

Barrero A, Ganan-Calvo AM, Davila J, Palacio A, Gomez-Gonzalez E (1998) Low and high Reynolds number flows inside Taylor cones. Phys Rev E 58(6):7309-7314

Chen YJ, Abbaschian R, Steen PH (2003) Thermocapillary suppression of the Plateau-Rayleigh instability: a model for long encapsulated liquid zones. J Fluid Mech 485:97-113

Chung AJ, Kim D, Erickson D (2008) Electrokinetic microfluidic devices for rapid, low power drug delivery in autonomous microsystems. Lab Chip 8(2):330-338

Dollet B, van Hoeve W, Raven JP, Marmottant P, Versluis M (2008) Role of the channel geometry on the bubble pinch-off in flowfocusing devices. Phys Rev Lett 1(3):034504

Fujimura T, Ikeda A, Etoh S, Hattori R, Kuroki Y (2003) Fabrication of open-top microchannel plate using deep X-ray exposure mask made with silicon on insulator substrate. Jpn J Appl Phys 42(6B):4102-4106

Ganan-Calvo AM (1998) Generation of steady liquid microthreads and micron-sized monodisperse sprays in gas streams. Phys Rev Lett 80(2):285-288

Ganan-Calvo AM, Gordillo JM (2001) Perfectly monodisperse microbubbling by capillary flow focusing. Phys Rev Lett 87(27):274501

Ganan-Calvo AM, Riesco-Chueca P (2006) Jetting-dripping transition of a liquid jet in a lower viscosity co-flowing immiscible liquid: the minimum flow rate in flow focusing. J Fluid Mech 553:75-84

Ganan-Calvo AM, Perez-Saborid M, Lopez-Herrera JM, Gordillo JM (2004) Steady high viscosity liquid micro-jet production and fiber spinning using co-flowing gas conformation. Eur Phys J B 39(1):131-137

Garstecki P, Gitlin I, DiLuzio W, Whitesides GM, Kumacheva E, Stone HA (2004) Formation of monodisperse bubbles in a microfluidic flow-focusing device. Appl Phys Lett 85(13): 2649-2651

Garstecki P, Fuerstman MJ, Stone HA, Whitesides GM (2006) Formation of droplets and bubbles in a microfluidic T-junctionscaling and mechanism of break-up. Lab Chip 6(3):437-446

Gordillo JM, Perez-Saborid M, Ganan-Calvo AM (2001) Linear stability of co-flowing liquid-gas jets. J Fluid Mech 448:23-51

Gunther A, Jensen KF (2006) Multiphase microfluidics: from flow characteristics to chemical and materials synthesis. Lab Chip 6(12): 1487-1503

Hibara A, Tsukahara T, Kitamori T (2009) Integrated fluidic systems on a nanometer scale and the study on behavior of liquids in small confinement. J Chromatogr A 1216(4):673-683

Kralj JG, Schmidt MA, Jensen KF (2005) Surfactant-enhanced liquidliquid extraction in microfluidic channels with inline electricfield enhanced coalescence. Lab Chip 5(5):531-535

Lam P, Wynne KJ, Wnek GE (2002) Surface-tension-confined microfluidics. Langmuir 18(3):948-951 
Link DR, Anna SI, Weitz DA, Stone HA (2004) Geometrically mediated breakup of drops in microfluidic devices. Phys Rev Lett 92(5):545031

Lo YC, Huang SS, Hsu WY, Wang C (2004) Neural guidance by open-top SU-8 microfluidic channel. In: Proceedings of the 2004 international conference on MEMS, NANO and smart system (ICMENS 2004), pp 671-674

Losey MW, Jackman RJ, Firebaugh SL, Schmidt MA, Jensen KF (2002) Design and fabrication of microfluidic devices for multiphase mixing and reaction. J Microelectromech Syst 11(6):709-717

Lu HP, Xun LY, Xie XS (1998) Single-molecule enzymatic dynamics. Science 282(5395):1877-1882

Malloggi F, Pannacci N, Attia R, Monti F, Mary P, Willaime H, Tabeling P, Cabane B, Poncet P (2010) Monodisperse colloids synthesized with nanofluidic technology. Langmuir 26(4): 2369-2373

Martín-Banderas L, Flores-Mosquera M, Riesco-Chueca P, Gil AR, Cebolla Á, Cháve S, Gañán-Calvo AM (2005) Flow Focusing: a versatile technology to produce size-controlled and specificmorphology microparticles. Small 1(7):688-692

Nakano M, Komatsu J, Matsuura S, Takashima K, Katsura S, Mizuno A (2003) Single-molecule PCR using water-in-oil emulsion. J Biotechnol 102(2):117-124

Nilson RH, Tchikanda SW, Griffiths SK, Martinez MJ (2006) Steady evaporating flow in rectangular microchannels. Int J Heat Mass Transf 49(9-10):1603-1618

Piorek BD, Lee SJ, Santiago JG, Moskovits M, Banerjee S, Meinhart CD (2007) Free-surface microfluidic control of surface-enhanced Raman spectroscopy for the optimized detection of airborne molecules. PNAS 104(48):18898-18901

Prakash M, Gershenfeld N (2007) Microfluidic bubble logic. Science 315(5813):832-835

Ravigururajan TS (1998) Impact of channel geometry on two-phase flow heat transfer characteristics of refrigerants in microchannel heat exchangers. J Heat Transf Trans ASME 120(2):485-491

Shui LL, Eijkel JCT, van den Berg A (2007) Multiphase flow in microfluidic systems - control and applications of droplets and interfaces. Adv Colloid Interface 133(1):35-49

Shui LL, Kooij ES, Wijnperle D, van den Berg A, Eijkel JCT (2009a) Liquid crystallography: 3D microdroplet arrangements using microfluidics. Soft Matter 5(14):2708-2712

Shui LL, van den Berg A, Eijkel JCT (2009b) A device for two-phase flow control in nanochannels. In: The 13th international conference on miniaturized systems for chemistry and life sciences (Micro total analysis systems- $\mu$ TAS2009), Jeju, Korea, pp 1509-1511

Shui LL, van den Berg A, Eijkel JCT (2009c) Interfacial tension controlled $\mathrm{W} / \mathrm{O}$ and $\mathrm{O} / \mathrm{W}$ 2-phase flows in microchannel. Lab Chip 9(6):795-802

Sinton D (2004) Microscale flow visualization. Microfluid Nanofluid $1(1): 2-21$
Skurtys O, Aguilera JM (2008) Applications of microfluidic devices in food engineering. Food Biophys 3(1):1-15

Sugiura S, Nakajima M, Seki M (2002a) Effect of channel structure on microchannel emulsification. Langmuir 18(15):5708-5712

Sugiura S, Nakajima M, Seki M (2002b) Prediction of droplet diameter for microchannel emulsification. Langmuir 18(10): 3854-3859

Tamaki E, Hibara A, Kim HB, Tokeshi M, Kitamori T (2006) Pressure-driven flow control system for nanofluidic chemical process. J Chromatogr A 1137(2):256-262

Tice JD, Lyon AD, Ismagilov RF (2004) Effects of viscosity on droplet formation and mixing in microfluidic channels. Anal Chim Acta 507(1):73-77

Tien J, Nelson CM, Chen CS (2002) Fabrication of aligned microstructures with a single elastomeric stamp. PNAS 99(4): $1758-1762$

Tseng FG, Lin KH, Hsu HT, Chieng CC (2004) A surface-tensiondriven fluidic network for precise enzyme batch-dispensing and glucose detection. Sens Actuator A 111(1):107-117

Utada AS, Fernandez-Nieves A, Stone HA, Weitz DA (2007) Dripping to jetting transitions in coflowing liquid streams. Phys Rev Lett 99(9):094502

van Dam DB, Le Clerc C (2004) Experimental study of the impact of an ink-jet printed droplet on a solid substrate. Phys Fluids 16(9):3403-3414

van den Berg A, Lammerink TSJ (1998) Micro total analysis systems: microfluidic aspects, integration concept and applications, vol 194. Microsystem technology in chemistry and life science. Springer, Berlin, p 33

van der Zwan E, Schroen K, Boom R (2009) A geometric model for the dynamics of microchannel emulsification. Langmuir 25(13): $7320-7327$

van Dijke KC, Schroen KCPGH, Boom RM (2008) Microchannel emulsification: from computational fluid dynamics to predictive analytical model. Langmuir 24(18):10107-10115

van Dijke K, de Ruiter R, Schroen K, Boom R (2010) The mechanism of droplet formation in microfluidic EDGE systems. Soft Matter 6(2):321-330

Wang KG, Yue SL, Wang L, Jin AZ, Gu CZ, Wang PY, Feng YC, Wang YC, Niu HB (2006) Manipulating DNA molecules in nanofluidic channels. Microfluid Nanofluid 2(1):85-88

Xu JH, Li SW, Lan WJ, Luo GS (2008) Microfluidic approach for rapid interfacial tension measurement. Langmuir 24(19): 11287-11292

Yang ID, Chen YF, Tseng FG, Hsu HT, Chieng CC (2006) Surface tension driven and 3-D vortex enhanced rapid mixing microchamber. J Microelectromech Syst 15(3):659-670

Yong Z, Novak R, Shuga J, Smith MT, Mathies RA (2010) Highperformance single cell genetic analysis using microfluidic emulsion generator arrays. Anal Chem 82(8):3183-3190 